\title{
Robots to assist daily activities: views of older adults with Alzheimer's disease and their caregivers
}

\author{
Rosalie H. Wang, ${ }^{1,2}$ Aishwarya Sudhama, ${ }^{3}$ Momotaz Begum, ${ }^{4}$ Rajibul Huq ${ }^{5}$ \\ and Alex Mihailidis', \\ ${ }^{1}$ Intelligent Assistive Technology and Systems Lab, Dept. of Occupational Science E Occupational Therapy, University of Toronto, Toronto, Ontario M5G \\ 1V7, Canada \\ ${ }^{2}$ Artificial Intelligence and Robotics in Rehabilitation Research Team, Toronto Rehabilitation Institute - University Health Network, Toronto, Ontario M5G \\ 2A2, Canada \\ ${ }^{3}$ Centre for Vision Research, York University, Toronto, Ontario M37 1P3, Canada \\ ${ }^{4}$ Department of Computer Science, University of New Hampshire, Kingsbury Hall, N215A, Durham, New Hampshire 03824, USA \\ ${ }^{5}$ The Mathworks, Natick, Massachusetts 01760, USA
}

\section{ABSTRACT}

Background: Robots have the potential to both enable older adults with dementia to perform daily activities with greater independence, and provide support to caregivers. This study explored perspectives of older adults with Alzheimer's disease $(\mathrm{AD})$ and their caregivers on robots that provide stepwise prompting to complete activities in the home.

Methods: Ten dyads participated: Older adults with mild-to-moderate AD and difficulty completing activity steps, and their family caregivers. Older adults were prompted by a tele-operated robot to wash their hands in the bathroom and make a cup of tea in the kitchen. Caregivers observed interactions. Semi-structured interviews were conducted individually. Transcribed interviews were thematically analyzed.

Results: Three themes summarized responses to robot interactions: contemplating a future with assistive robots, considering opportunities with assistive robots, and reflecting on implications for social relationships. Older adults expressed opportunities for robots to help in daily activities, were open to the idea of robotic assistance, but did not want a robot. Caregivers identified numerous opportunities and were more open to robots. Several wanted a robot, if available. Positive consequences of robots in caregiving scenarios could include decreased frustration, stress, and relationship strain, and increased social interaction via the robot. A negative consequence could be decreased interaction with caregivers.

Conclusions: Few studies have investigated in-depth perspectives of older adults with dementia and their caregivers following direct interaction with an assistive prompting robot. To fulfill the potential of robots, continued dialogue between users and developers, and consideration of robot design and caregiving relationship factors are necessary.

Key words: activities of daily living, aged care, Alzheimer's disease, carers

\section{Introduction}

Alzheimer's disease (AD) and other dementias can result in decreased attention, memory loss, poor executive functioning, and changes in language and comprehension (American Psychiatric Association, 2013). Older adults with $\mathrm{AD}$ and other dementias often require support from family caregivers

Correspondence should be addressed to: Rosalie Wang, Intelligent Assistive Technology and Systems Lab, Department of Occupational Science and Occupational Therapy, University of Toronto, 160-500 University Avenue, Toronto, Ontario M5G 1V7, Canada. Phone: +1-416-946-8566. Email: rosalie.wang@utoronto.ca. Received 7 Jun 2016; revision requested 16 Jul 2016; revised version received 6 Aug 2016; accepted 8 Aug 2016. First published online 23 September 2016. to perform daily activities. Difficulties manifest in initiating tasks, problem solving, executing activity sequences, and balancing safety concerns. Maintaining abilities to perform meaningful activities is desirable in order to preserve feelings of independence and well-being. For example, completing everyday activities such as making a cup of tea autonomously is important as it can provide a sense of social significance, promote feelings of accomplishment and presence, support learning and engagement, and foster positivity (Sakuae and Reid, 2012).

Family caregivers often strive to preserve older adults' sense of autonomy; dementia caregiving 
can provide positive opportunities for the caregiver like becoming closer to the care receiver, and provide a sense of reciprocity (Peacock et al., 2010). However, the majority of family caregivers $(68 \%)$ of persons with dementia experience burden and stress from caregiving, and 65\% experience depressive symptoms (Papastavrou et al., 2007). Caregivers face day-to-day challenges in supporting physical safety and daily activity performance as ongoing supervision and step-by-step reminders become necessities. The need for assistance is compounded when older adults have concurrent physical limitations. Feelings of burden and stress associated with caring for persons with dementia are linked to mental exertion, feelings of depression, physical exertion, and risk of cardiovascular disease (Papastavrou et al., 2007; Richardson et al., 2013; Jennings et al., 2015). Options to augment care with technology, possibly with the use of robots to assist persons with dementia to perform daily activities (Faucounau et al., 2009; Kerssens et al., 2015), can contribute to improvements in quality of care and decrease caregiver burden and stress (Mitzner et al., 2010).

Assistive robots can help people physically (e.g., retrieving items) and/or socially (e.g., providing encouragement). Robots are being explored worldwide to help older adults in various ways (Bedaf et al., 2015). For example, the European Union CompanionAble project aimed to assist persons with mild cognitive impairment and included a multifunctional mobile robot Hector that communicated with a smart home environment (Schroeter et al., 2013). Among other features of the integrated system, Hector provided high-level reminders for appointments based on the system's stored agenda, suggested predetermined activities to be carried out, offered a means for video chats with others, and provided cognitive stimulation activities. Remotely-controlled mobile robots such as Giraff have been investigated with older adult long-term care residents with mild-to-moderate dementia in Australia (Moyle et al., 2014). The purpose of the robot was to allow video chats between older adults and their family, thereby supporting social interaction and reducing isolation. While most assistive robots remain in development, robotic pets, such as Paro the baby harp seal, are commercially available. Developed in Japan, Paro was intended to fulfill psychosocial and companionship needs of older adults with severe dementia in hospitals and long-term care homes (Shibata, 2012). Paro, the most studied robot for older adults with dementia, has been found in studies in multiple countries to improve mood and decrease stress with residents, foster communication between residents, and even decrease staff stress.
Few robots, however, have been developed to assist older adults with dementia who require stepby-step prompting to complete everyday activities. While various low and high technologies have been applied (e.g., large format boards with reminders) or developed (e.g., mobile applications for smart phones), for some older adults who require stepwise activity prompting, a satisfactory solution has yet to be found. Some environmentally embedded technologies (e.g., used with smart homes) have explored stepwise prompting strategies for older adults with dementia (e.g., for drinking water, brushing teeth and upper body dressing (Bewernitz et al., 2009), and handwashing (Mihailidis et al., 2007 ; 2008). While they are designed to require little interaction from users to initiate assistance, embedded systems cannot adequately engage users with their static interfaces and cannot track and assist users when they move away from locations where the sensors and interfaces are installed. A mobile, interactive robot interface that can provide stepwise prompts may address these issues for applications in the home. A robot's embodied physical presence can help to make interactions more socially engaging, effective, and acceptable. Previous studies have described the benefits of providing instructions through a robot compared to a tablet computer (Mann et al., 2015) and a virtual coach (Fasola and Matarić, 2013). The social presence of the robot and its nature may increase engagement with the robot and motivation to comply with directions.

To develop robots that will be acceptable and useful to older adults with $\mathrm{AD}$ and other dementias and their caregivers, it is essential to understand and incorporate users' perceptions, attitudes, and needs regarding use of robots in daily life at home (Faucounau et al., 2009; Mitzner et al., 2010). Older adults need to be more involved during the design process so that their needs and concerns are addressed (Frennert and Ostlund, 2014) and there is a significant gap in assistive robotics research examining the perspectives of older adults with $\mathrm{AD}$ and their caregivers. It is rare to find studies that present the detailed views of older adult users with $\mathrm{AD}$ along with their caregivers' perceptions, and even fewer studies that report this information following direct interaction with an assistive robot. Most studies investigating the role of robotic assistants for home use employ surveys and questionnaires, which provide a broad understanding of older adult and caregiver perceptions. The disadvantage of this methodology is the lack of in-depth exploration of varying perceptions and possible biases inherent in the questions being asked. Indepth examination through qualitative interviews 


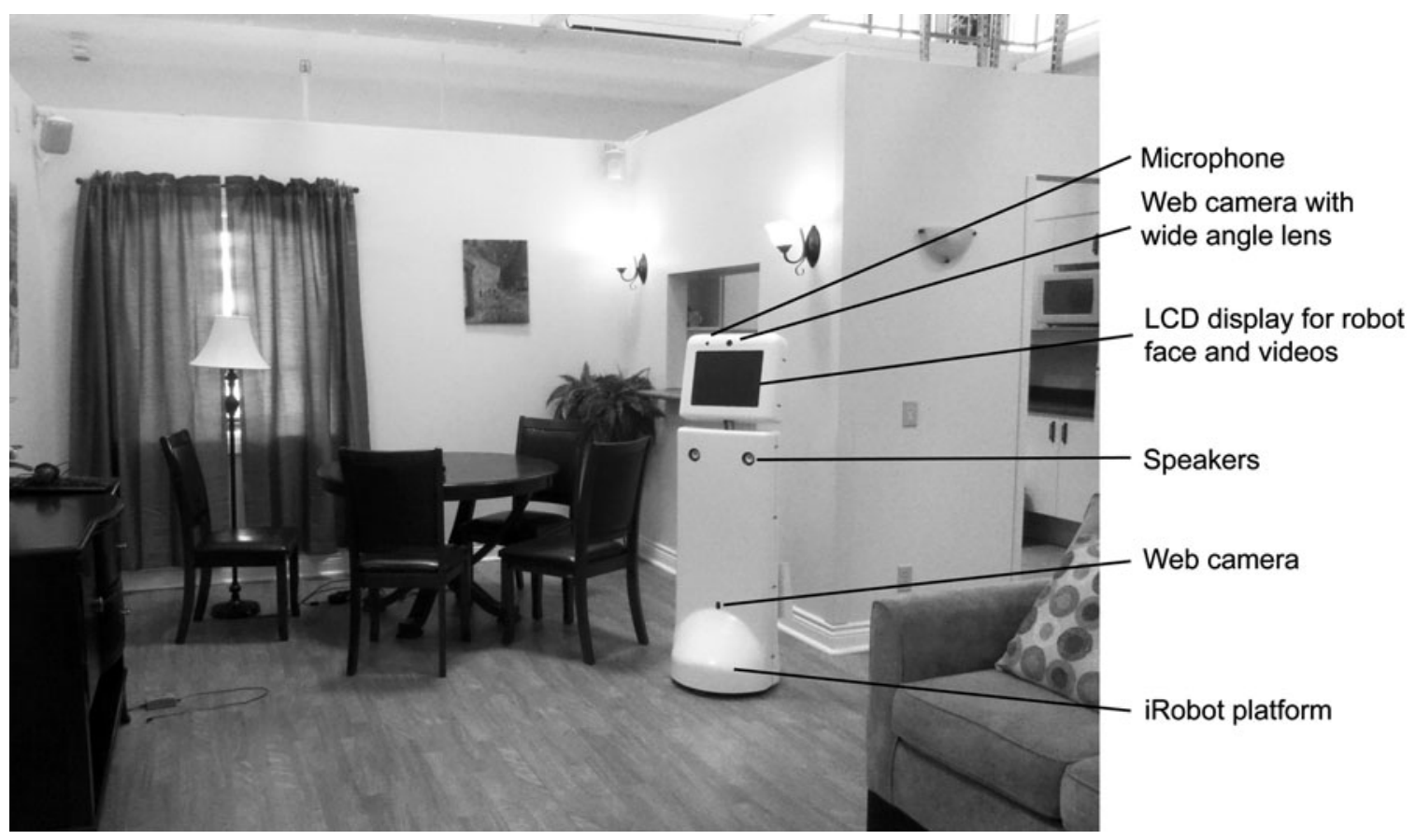

Figure 1. Tele-operated assistive robot, "Ed", inside HomeLab, indicating components of robot.

and analysis following interaction with a robot can provide developers with indispensable knowledge in regards to supporting acceptance, utility, and higher integration of robotic assistants within the home. This paper addresses the following questions with respect to older adults with $\mathrm{AD}$ and their caregivers:

1. What are the perceptions of an assistive robot that provides step-by-step assistance for daily activities?

2. What are the implications for social relationships with the introduction of an assistive robot in the caregiving context?

\section{Methods}

\section{Study design}

This paper included qualitative, thematic analysis of semi-structured interview data from older adults with mild-to-moderate $\mathrm{AD}$ and their caregivers collected from a descriptive mixed methods study. The main study examined the usability of a robot that provided step-by-step prompting assistance to complete activities, in this case hand washing and tea making. A descriptive mixed methods approach was used for the study given the limited information available on assistive robot use by these user groups and the need to elucidate design requirements to guide technical development. Data from the main study, not included in this analysis, consisted of audio/video recordings of the older adults' robot interactions (to enable behavioral observation coding and transcriptions of verbal interactions) and questionnaires. The interview guides were developed by the research team, which included researchers with clinical and research experience working with older adults with dementia. The guides were also reviewed by two expert researchers prior to use in the study.

\section{Setting}

The study was conducted in HomeLab (iDAPT, Toronto Rehabilitation Institute), a simulated home (Figure 1). Approval was received from the research ethics boards of Toronto Rehabilitation Institute and the University of Toronto. Data were collected between August and December 2012.

\section{Participants}

Participants were recruited from clinical partners at Toronto Rehabilitation Institute sites and local community-based care agencies, and through advertisements posted at clinical sites and non-profit organizations (e.g., Alzheimer Society of Canada). Clinical partners screened participants according to the inclusion/exclusion criteria. If participants were eligible, the clinical partner set up appointment times for the participants and researchers. If responding to an advertisement, participants could call the researchers to enquire about the study. Participants were enrolled consecutively.

Older adults and their caregivers were recruited as dyads. Older adults had to be 55 years or older, able to hear normal levels of speech, diagnosed 


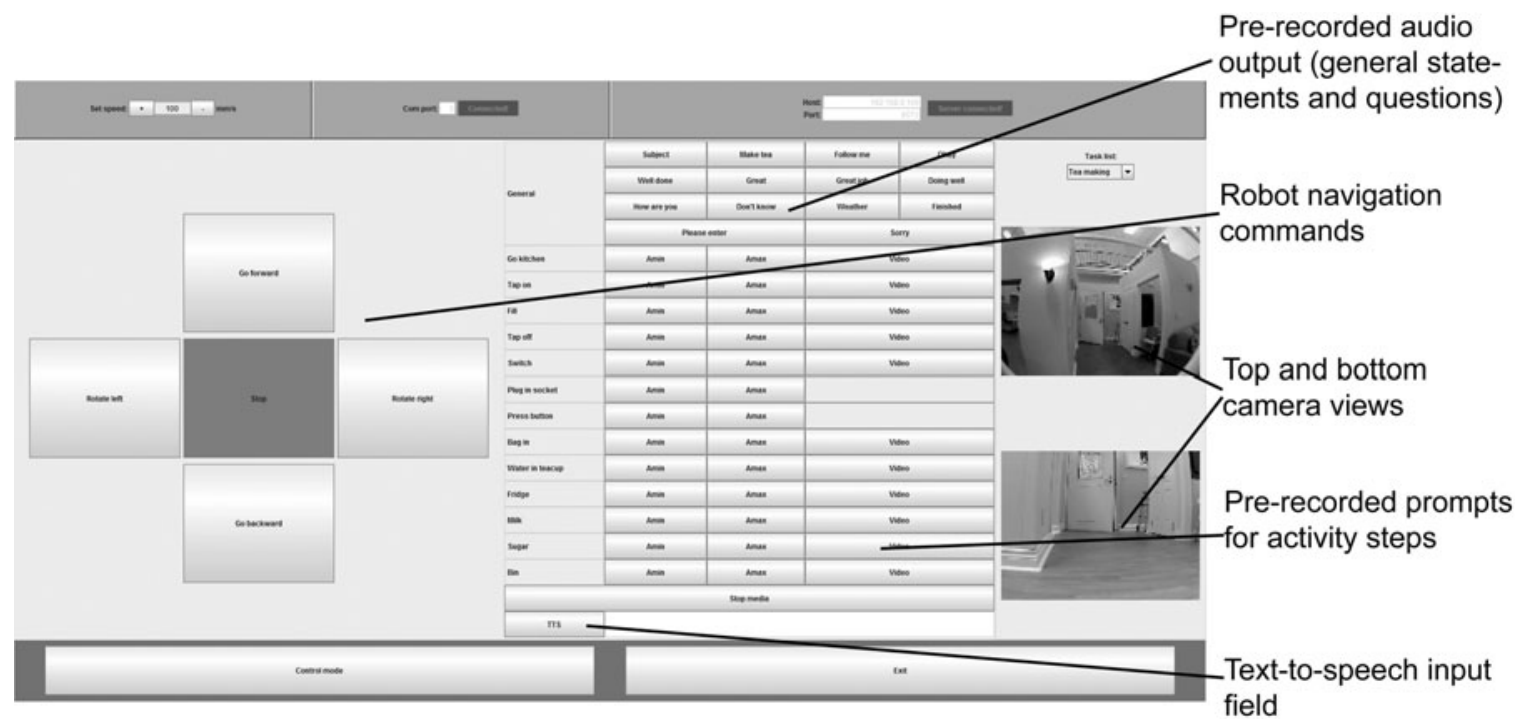

Figure 2. Display of tele-operator's screen, indicating commands available to the operator during the study.

by their physician as having mild-to-moderate $\mathrm{AD}$, experiencing difficulty completing common sequences of activity steps (according to their caregiver), and able to give informed consent or assent (with a substitute decision maker giving consent). Caregivers had to be 19 years old or older, a family member or privately hired caregiver who provides more than seven hours of care per week, and able to give informed consent. All participants had to be fluent in English.

Five to ten dyads were targeted for recruitment. Nielsen and Landauer (1993) suggested that five participants are sufficient to identify a majority of the usability issues before moving to the next design iteration for evaluation. As such, data from 5-10 dyads was anticipated to be sufficient to explore the research questions in the main study.

\section{Assistive robot, "Ed"}

A custom-designed tele-operated robot, Ed, was used. A trained tele-operator remotely controlled Ed's movements, speech, and prompting functions. Ed was constructed with an iRobot Create platform (iRobot, Boston, USA), a personal computer, a LCD screen, microphones, speakers, and web cameras inside a customized shell (Figure 1). The LCD screen displayed either a face (with lips that moved during speech) or pre-recorded videos. Audio output included both pre-recorded (e.g. statements, questions, and prompts) and realtime audio output via the tele-operator interface (Figure 2). All audio output was via a text-tospeech synthesizer (Cepstral, "David" voice).

\section{Procedures}

Following consent procedures the Mini Mental State Examination (Folstein et al., 1975) was administered to screen for dementia and for descriptive purposes. Demographic and background information was collected in an interview format with each dyad.

Participants were introduced to Ed and received a brief demonstration of Ed's functions (movement, communication, and prompting). Participants were informed that the researcher would operate Ed remotely. The older adult was oriented to HomeLab and asked to complete the two activities with Ed providing stepwise prompting, as needed. The two daily activities, hand washing in the bathroom and tea making in the kitchen, were broken down into several prompt steps (Figure 3). Audio and audiovisual prompts were formulated according to these steps. Each of the prompts were prerecorded and delivered with an increasing degree of assistance. This systematic method ensured efficiency of the prompting method. Prompting assistance progressed as follows: Min - suggestive audio prompt (e.g., "Can you fill the kettle with water now?"), Max - directive audio prompt, preceded by the participant's name (e.g., "Try filling the kettle under the water"), and MaxVid Max prompt with a video clip demonstrating how the step is performed (e.g., model picks up kettle and puts it under tap with running water). The audio and audiovisual prompts were similar in type and format to those used in our previous studies with the COACH (Cognitive Orthosis for Assisting aCtivities in the Home) system (Mihailidis et al., 2007; 2008). Because Ed was mobile, guidance was provided for activity locations and by whole body gestures that directed attention to items within the environment.

Caregivers were asked to observe the interaction with Ed, and to provide feedback on their 


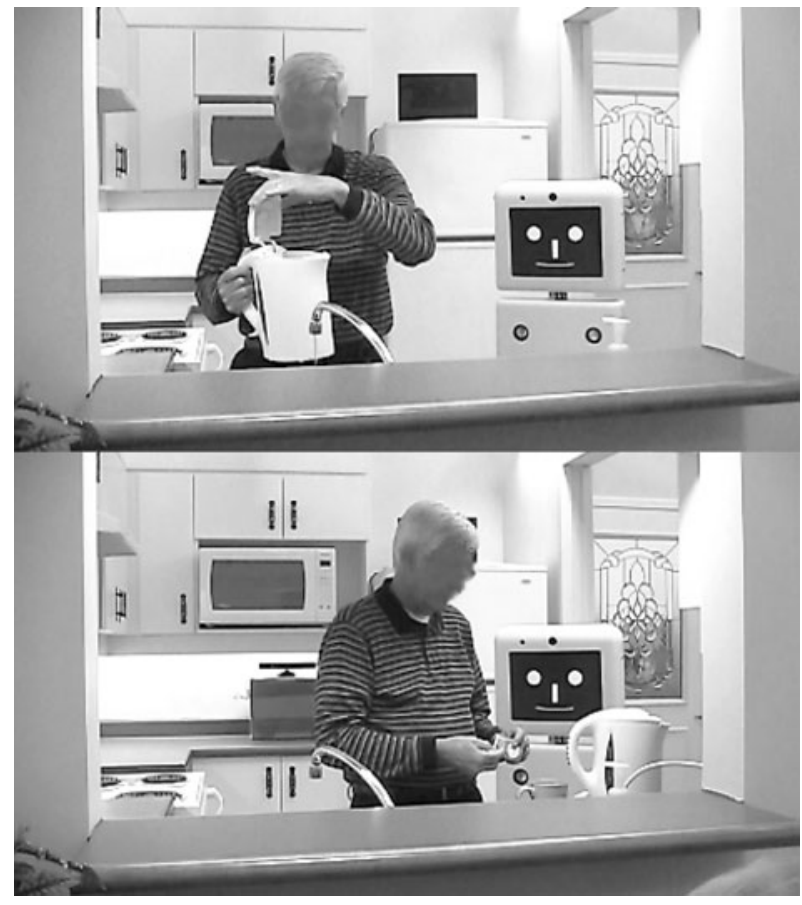

Figure 3. Tele-operated assistive robot, "Ed", assisting a participant to make a cup of tea in the kitchen.

observations afterwards. They were asked to refrain from interacting with the older adult, unless they felt the older adult was upset, frightened, or uncomfortable. A researcher was present at all times to monitor the older adults' responses and to intervene if necessary (e.g., for personal or technical assistance). Older adults and caregivers completed semi-structured interviews (and questionnaires) separately. Interviews with the older adults were conducted with the robot nearby to allow reference to the robot as needed. The semi-structured interviews, while including specific and concrete questions intended to be easily understood, were also flexible to allow interviewers to ask further questions to explore ideas brought up by the participants. The interview guides for the older adults and caregivers are included in appendix A1 (published as supplementary material online attached to the electronic version of this paper at http://journals.cambridge.org/ipg). Audio and video recordings were made throughout each study session.

\section{Qualitative data analysis}

Interviews were transcribed verbatim and reviewed iteratively by two authors (RHW and AS). Thematic analysis was conducted with the transcripts to gain insight into how participants perceived their experiences with the robot. Thematic analysis allowed for categorization of large amounts of data so that they can be interpreted, enabled pattern identification, and made it easier to tease apart consistencies and variations within and between the groups (Saunders and Byrne, 2002; Braun and Clarke, 2006). Initially, the two authors familiarized themselves with the individual interviews for the older adults and the caregivers. Data were examined by dyads and within groups to explore themes beyond comparisons of the two groups. The authors individually reviewed and developed preliminary codes for three sets of dyad interviews ( $30 \%$ of randomly selected sets). The authors reviewed each transcript together to discuss individual codes. Assumptions and differences in interpretations were discussed until agreement. The coding scheme was refined and preliminary categories for the data were defined. Both authors individually and then together reviewed the other transcripts to refine the codes and categories. Categories were then organized into a thematic map with themes and subthemes. Continued analysis with the other authors to explore alternative understandings of the data helped to consolidate the themes. Segments of transcripts that best represented the themes and subthemes were included in the manuscript as illustrative examples.

Several strategies were used to ensure trustworthiness (Saumure and Given, 2008) in the data collection and analysis. The authors who conducted the interviews (RHW and MB) and other members of the research team debriefed following each dyad's study session to reflect on initial observations and interview findings. Strategies to refine interview questions and techniques were discussed in preparation for subsequent study sessions. To enhance dependability and credibility, two authors analyzed the data. The two authors also reviewed, compared, and discussed individual cases to examine those that supported or challenged emerging ideas. Finally, the diverse backgrounds of the researchers served as a form of triangulation to examine biases and alternative approaches to understanding the data. The authors have complementary backgrounds in occupational therapy, biology, psychology, robotics, and biomedical engineering.

\section{Results}

\section{Description of participants}

Ten dyads were recruited from one community care agency. Dyads were numbered, where OA represented the older adult and $\mathrm{C}$ represented the caregiver. Table 1 summarizes the demographic information of participants. OA 1 and $\mathrm{C} 1$ were unable to complete the full interviews because of 
Table 1. Participant demographics

\begin{tabular}{|c|c|c|c|c|c|c|c|}
\hline OLDER ADULT & AGE & GENDER & $\mathrm{MMSE}^{1}$ & LIVES ALONE $(\mathrm{Y} / \mathrm{N})$ & CAREGIVER & AGE & RELATIONSHIP \\
\hline OA1 & 76 & $\mathrm{~F}$ & 9 & $\mathrm{~N}$ & $\mathrm{C} 1$ & 36 & Son \\
\hline OA2 & 86 & $M$ & 24 & $\mathrm{~N}$ & $\mathrm{C} 2$ & 76 & Wife \\
\hline OA3 & 88 & $M$ & 25 & $\mathrm{~N}$ & C3 & 58 & Daughter \\
\hline OA4 & 77 & $\mathrm{~F}$ & 25 & $\mathrm{Y}^{3}$ & $\mathrm{C} 4$ & 48 & Daughter \\
\hline OA5 & 59 & $\mathrm{~F}$ & $18^{2}$ & $\mathrm{~N}$ & C5 & 59 & Husband \\
\hline OA6 & 63 & $M$ & 23 & $\mathrm{~N}$ & C6 & 63 & Wife \\
\hline OA7 & 77 & $\mathrm{~F}$ & $25^{2}$ & $\mathrm{~N}$ & $\mathrm{C} 7$ & 78 & Husband \\
\hline OA8 & 83 & $\mathrm{~F}$ & $19^{2}$ & $\mathrm{~N}^{4}$ & $\mathrm{C} 8$ & 56 & Son \\
\hline OA9 & 84 & $\mathrm{~F}$ & 25 & $\mathrm{Y}$ & C9 & 54 & Daughter \\
\hline OA10 & 85 & $M$ & 15 & $\mathrm{~N}$ & $\mathrm{C} 10$ & 74 & Wife \\
\hline
\end{tabular}

${ }^{1}$ MMSE: Mini Mental State Examination.

${ }^{2}$ Item for spelling "WORLD" backwards as opposed to serial counting.

${ }^{3}$ With dog.

${ }^{4}$ In separate suite of son and family's home.

Table 2. Overview of themes and subthemes

\begin{tabular}{lll}
\hline THEMES & & SUBTHEMES \\
1 & Contemplating a future with assistive robots & Impressions \\
2 & Considering opportunities with assistive robots & Robots for home use \\
3 & Reflecting on implications for social relationships & Influence on caregiving dynamics \\
& & A robot's role: Friend or appliance? \\
\hline
\end{tabular}

time limitations and OA1 was also fatigued and had difficulty responding to all of the questions.

\section{Summary of themes and subthemes}

Table 2 summarizes results from the thematic analysis presented as themes and subthemes.

\section{Theme 1: Contemplating a future with assistive robots}

IMPRESSIONS

Both older adults and caregivers were captivated by the novelty of the robot. Reflecting on her experience with Ed, OA7 exclaimed: "Oh I was fascinated!...it's coming towards you...it's getting messages...it's almost like watching something on $T V$... the whole thing has been great." C6 relayed her story of hearing about study: "...when they called us to see if we'd be interested ....to work with a robot, I kind of laughed, I thought that sounded crazy...having seen it work... I'm not thinking it's crazy anymore...You sold me."

Participants wanted to learn more about robots out of curiosity, but also wanted more information or exposure before considering a robot in their lives. OA2 wondered about reliability and maintenance needs. Caregivers posed specific questions on the robot's development, adaptability, and appropriateness for varying users. C9 asked about the robot's suitability for users with varying cognitive levels and questioned if the robot could adapt to users' preferences and changing abilities. C10 wondered about cost-effectiveness, how long a person with $\mathrm{AD}$ might make use of a robot, and also whether it may be reprogrammed for someone else if the original owner no longer needed it. Overall, the experience was thought-provoking and novel for participants and many had not considered such a possibility prior to this study.

\section{ROBOTS FOR HOME USE}

Older adults' views were mixed with regards to having robots assist in their daily lives. Some did not want a robot (e.g., OA1, OA10), others were unable to respond (although appeared to enjoy the interaction, e.g., OA5, OA6), and the remaining were open to robotic assistance. Most participants felt they did not need one, but considered it as a future possibility. Primary reasons for not wanting a robot were because they lived with a family member who helped them or they did not feel they were at a stage where a robotic assistant was necessary. The two older adults with mild dementia and who lived alone (OA4 and OA9) were among those who did not feel a robot was needed. Other reasons for not wanting a robot at the time included psychological readiness (e.g., OA7 was not prepared to have someone in addition to her husband reminding her of things), preference for assistance from an "efficient human being" (OA9), challenges in 
learning new skills, and the robot's questionable cost-effectiveness (OA10). Older adults suggested a robot would be useful for someone living alone, who did not have help, or whose health, primarily physical health, they perceived as worse off than their own. OA4 indicated that a robot would be worthwhile later if it prevented a move to a care home and reduced her reliance on her children.

The caregivers' openness and willingness toward robot use diverged from the older adults' with regard to the timing of having a robot. Most were open to the possibility of using assistive robots and several (C3, C4, C6) would use a robot right away. C6 especially welcomed the possibility: “...I think it ['d] be fun to take it home with me and try different things...just to see, which ones it would work with and which chores it wouldn't and see how [OA6] would respond to it...I need all the help I can get believe me...." C4 enthusiastically stated, "Work faster so then we can get them in the houses for everybody...I thought it was awesome." Caregivers' openness toward robot use did not differ when considering the older adults' stage of dementia or whether the older adults lived alone. Interestingly, some caregivers incorrectly felt that the older adults would be just as open as they were to using and integrating a robot right away.

\section{Theme 2: Considering opportunities with assistive robots}

Regarding the two activities explored in this study, most participants did not feel that prompting assistance from a robot was necessary during hand washing, as this was a common activity. Tea-making in contrast was a more complex and unfamiliar activity, and so prompting from a robot was seen as beneficial.

Direct interaction with the robot stimulated much consideration for other daily activities in which reminders and prompting assistance may be useful. Caregivers tended to provide more activity examples and tended to be more specific in their suggestions compared to the older adults. Caregivers often focused on needing support to ensure older adults' physical safety (e.g., detection of falls, or reminders for locking doors, or turning off the stove), but in contrast, none of the older adults mentioned needing safety reminders. The robot was acknowledged by some of the older adults and caregivers to be applicable to assisting older adults to complete unfamiliar activities (e.g., for OA4 - making tea) and reinforcing routines (e.g., for C6 - getting up in the morning, weighing himself, taking blood pressure). Examples of opportunities ranged from reminders of general information (e.g., appointments, orientation to time, locations of items, safety precautions) to assistance with basic activities of daily living (e.g., dressing, showering) and more complex instrumental activities of daily living (e.g., paying bills, taking medications, preparing meals, doing laundry). Caregivers mentioned instrumental activities of daily living such as cooking and cleaning to be important to facilitate with older adults, as they often wished to contribute to household activities. The assistance levels ranged from highlevel reminders to stepwise prompts, as caregivers noted many activities necessitated detailed step-bystep prompting because of uninitiated, missed, or out of sequence steps.

The varied examples and expressed needs highlighted potential benefits of using embodied systems (e.g. mobile interactive robot with a social presence) as opposed to static or embedded systems (e.g. notes, reminder boards or even embedded sensors and screen interfaces). Older adults carried out activities in different locations in the home where context-specific and often dynamic and tailored reminders or prompts were required. C7 suggested using a giant memo device with the day's events or leaving notes but he, along with other caregivers, recognized that these aids were not consistently or effectively used. For example, older adults had to remember to go to a specified location to get required information. Once there they may not remember the context of the needed information. C6 noted how readily OA6 conversed with the robot and felt that he would enjoy engaging with a social robot that provided him with needed orientation and activity information and thereby minimize confusion and frustration. She noted the usefulness of such a robot: "... if I could program that robot at the beginning of everyday just to repeat what day it is, what month it is, what the activities are for that day... if I had a robot that just could repeat every ten minutes, he could push the button and the robot would repeat to him today is...." C8 mentioned that a robot could function to reduce anxiety and to provide answers to repetitive questions; the social presence of the robot could induce feelings of comfort for the older adult. The interactivity and social presence of a robot may be useful for complex activities that required older adults to make context-specific choices. Dressing was identified by several caregivers to be a challenging activity because of the choices that need to be made to select suitable clothing (e.g., to put on a clean shirt or event-appropriate clothing) and the need to locate and sequence clothing to dress appropriately. C7 described the daily dressing process to be difficult because OA7 required stepwise prompting but refused family assistance. An interactive robot that prompted, 
offered positive encouragement along the way, and offered choices that respected her autonomy could better enable activity completion. C2 noted, however, that complex medication regimes or those requiring physical administration (e.g., eye drops) would likely not work, even with an embodied robot.

\section{Theme 3: Reflecting on implications for social relationships}

INFLUENCE ON CAREGIVING DYNAMICS

Experiences with the robot generated discussion around the potential impact of a robot on caregiving dynamics. Both older adults and caregivers gave examples showing the varied ways in which a robot may improve interactions and relationships by avoiding conflict, decreasing worry and anxiety, and moderating frustrating interactions that may have long term consequences. Some caregivers felt that if the robot provided accurate prompting assistance, they could focus their resources on other aspects of the relationship. OA4 stated that a robot might allow her to stay living at home longer and avoid family conflict in deciding with which of her children she would live. C6 and C8 illustrated the frustrating daily interactions they experienced and identified that a robot could reduce relationship strain. C6 stated: "...this is where your wallet is, this is where your keys are, those are the things that we just spend so much time going over and over....I just feel so repetitive and then I lose my patience and then I feel terrible...cause it's not his fault." She recognized that her husband was impacted by these interactions because he apologized for asking repetitive questions or he tried not to ask for help. She thought a robot may be a solution because asking a robot may be perceived as being less burdensome than asking her. C8 acknowledged the escalating and negative effects of frustrating interactions on their relationship: "...Those are actually the things that drive you crazy...It's difficult for them but they often are not the ones who are, you know frustrated by it... it's not good for them because, you get frustrated as the caregiver and they can see you're frustrated that you just answered the same question for th... $72^{\text {nd }}$ time...." $\mathrm{C} 8$ reported that his mother needed constant reassurance such that the family was unable to leave her alone: "...she gets anxious being by herself...Far as she knows we're gone forever..." After observing his mother speaking with the robot, C8 suggested that a robot might help reduce some anxiety and relationship strain. A robot companion could pacify some of her anxiety by answering her questions about where her family has gone, reminding her when they will return, and assisting by calling them if needed. A robot could also help with difficulties in finding a caregiver for two to three hours, alleviating stress for the whole family. C8 suggested that a robot might help his mother perform daily activities because “...she doesn't like it sometimes when we help her". He was encouraged because he saw that she readily asked questions of the robot about the next steps to carry out rather than wandering around looking confused, which was usual at home.

Worthy of mention was participant, OA7, who described a potentially strained relationship with her husband, and contrary to other participants' reports, she suggested that a robot might negatively disrupt her relationship with her husband. She was acutely aware of her declining abilities and valued the time they spend together doing household chores. She worried that he may want a robot so that he could spend more time alone with his computer at the expense of spending time with her.

A ROBOT'S ROLE: FRIEND OR APPLIANCE? The robot interactions stimulated deliberation on a robot's varied role in social relationships, with some notable consistencies and variations among older adults and caregivers. The role that a robot might assume ranged from a friend who supported social and emotional needs to a machine that enabled activity performance in the home with little capacity for personal connection. Most superficial were variations in how participants addressed the robot (i.e., "he" or "it") and the extent to which participants attributed human-like qualities to the robot. Many of the older adults, but none of the caregivers, showed ambivalent responses. OA3 stated that the robot was "an inanimate object" but recalled his conversation with the robot and determined that he was no longer sure since it could provide companionship for someone who was alone. OA8 who expressed some degree of social isolation spoke most compellingly of a robot being a valuable friend. The robot was "somebody" she could talk with, that she could tease, and that she did not resent. She enjoyed the exchange with the robot. The robot's presence might feel like there was someone in the house so there may "not [be] so many spaces of emptiness". Nevertheless, she said robots are "a necessity, but you can never replace people".

As many older adults as caregivers viewed the robot as a machine-like appliance. OA9 poignantly asserted the robot's place: “...[I was] doing what I was asked to do, and... instead of talking to a person I was talking to a machine.... it's still a machine... which a human being is not...." She added, “...you don't feel connected to it because it's a robot and you're not...." Similarly, C7 felt that the connection or 
attachment with people would not be there: 'I don't think anybody is going to become attached to it...even in the deepest of dementias I don't think anybody's going to suddenly go over and hug it and kiss it and say, that's my friend..." C10 was also convinced that the robot was a machine: “... it's a piece of machinery... just like your computer screen ...I just saw the two eyes the nose and... well it doesn't really make you responsive, emotion response, it's a piece of machinery."

\section{Discussion}

Qualitative analysis of the semi-structured interviews offered evidence to support the proposed practical and social benefits of robots to care for older adults with mild-to-moderate $\mathrm{AD}$ and to help caregivers. Robots to assist older adults with dementia by providing stepwise prompts are uncommon. Findings also revealed that while some caregivers were eager, older adults were open to the idea but not prepared to have robots assist them. This paper contributes findings to advance the field by presenting previously undocumented in-depth perspectives of older adults and their caregivers on whether they want a robot, opportunities they see with home robots, and the anticipated relationship consequences of robot use. The following discussion addresses the research questions in relation to the resultant themes, presents important considerations for assistive robot development and integration, and outlines the study's limitations.

\section{Perceptions and consistencies or variations in perceptions}

Older adults in this study, with a few exceptions, were curious and open to the idea of assistive robots. Other studies have shown that older adults respond positively towards robots after watching video demonstrations of a robot's capabilities such as delivering objects (Smarr et al., 2014) or live demonstrations of a robot with functions such as playing a memory card game (Louie et al., 2014). While open to the idea, none of the older adults in this study expressed the immediate desire to use one. Their reasons for not wanting a robot suggested a lack of perceived need for the robot's services. Others studies have demonstrated that older adults are accepting of assistive technology if benefits of use are clear (Mitzner et al., 2010). Participants in our study considered future robot use, if their health, especially physical health declined. The robot was deemed useful for others whose health was worse off than their own or who were socially isolated, suggesting that they perceived themselves to be more able or better cared for than the expected users. Similarly, Wu et al. (2016) found that older adults with mild cognitive impairment felt they did not need an assistive robot as it would be useful for those who were older or more disabled. These findings indicate that the social stigma often associated with assistive technology users being old or disabled extended to robot users. Alternatively, maintaining autonomy and independence is of utmost importance for older adults with dementia (Samsi and Manthrope, 2013) and this may translate to robot use. At least for OA4, if using a robot allowed her to live independently at home for longer, a robot would be a serious consideration.

Caregivers in this study were more open and accepting to having a robot sooner, and suggested more opportunities for activities in which robots may help compared to older adults. Daily challenges with care and management of older adults' $\mathrm{AD}$-related symptoms may have contributed to the differences in perspectives. Caregivers often experience the shortcomings of current strategies to assist in daily activity performance, maintenance of safety, and support for wellbeing. Studies of family caregivers of persons with dementia have suggested that technology may be useful to address many of these shortcomings (Broadbent et al., 2012; Rosenberg et al., 2012; Kerssens et al., 2015). In the study by Broadbent et al. (2012), caregivers mentioned that if a robot could do certain tasks (e.g., distributing meals), caregivers could have more time to spend with residents. Cognitive impairments in memory, abstract thinking, awareness of limitations, or familiarity with technology may also have hindered older adult participants from describing more opportunities.

\section{Implications for social relationships}

The second objective explored the implications of introducing a robot in the care of an older adult with $\mathrm{AD}$ and how this might impact social relationships. In a relationship-centered approach that is necessary in dementia care, it is imperative that assistive technology provide older adults with cognitive support and assistance to participate in daily activities, and provide caregivers with assurance and respite (Topo, 2009). Findings from this study indicate that there are differences in the views of older adults with mild-to-moderate $\mathrm{AD}$ and their caregivers toward robot use. A balance must be achieved to ensure that robots are recommended based on the older adults' and caregivers' needs and preferences, otherwise there is a risk for loss of autonomy, privacy, and dignity for the older adult. 
Participants indicated that robots may help older adults to carry out daily activities with less caregiver reliance and may decrease relationship strain. Some caregivers expressed worry, stress, exhaustion, and frustration owing to the older adults' agitation, anxiety, and repetitive questioning. These are known behavioral and psychological symptoms of dementia and arguably the most challenging symptoms for family caregivers to manage (Kales et al., 2015). Having robots take over frustrating or repetitive activities can free caregivers to do more valued activities with the older adult. With some participant dyads a robot may help to decrease relationship tension when an older adult with $\mathrm{AD}$ does not like receiving help from a caregiver or does not want to constantly ask for help. While the experience of caregiver stress results from multiple factors, further study is needed to ascertain whether a robot can moderate caregiving stress or increase the frequency of mutually positive experiences.

A critical concern with assistive robots includes implications for social contact. Social contact and support are important for well-being and older adults with dementia are at risk for experiencing loneliness (Moyle et al., 2011). OA7 highlighted a common concern of whether or not having an assistive robot decreases social contact (Sharkey and Sharkey, 2012) or results in neglect by family. In contrast, some older adult participants spoke spontaneously of the robot's potential to function as a social companion. Caregivers also value the caregiving role and so they may not want to be "replaced" by a robot (Vallor, 2011). Prevalent benefits of caregiving include having a stronger sense of companionship, developing a sense of reciprocity, fulfilling a duty/obligation, feeling an increased sense of competence and personal growth as one assumes a caregiving role, and having a sense of improving the quality of life for the care receiver (Cohen et al., 2002; Peacock et al., 2010). The variations in participants' responses highlight the necessity to understand the needs, preferences, and living situations of older adults and their caregivers if considering the inclusion of a robot in daily care.

\section{Considerations for assistive robot development and integration}

Several considerations emerged that will be necessary for the development of useful robots and the future integration of robots into the homes of older adults with $\mathrm{AD}$ and their caregivers.

1. Participants expressed general interest and desire to learn about robots for daily activities. It will be critical for researchers to pursue this dialogue with other older adults and caregivers in the context of public education and robot development to continue knowledge exchange. With knowledge and engagement, acceptance and integration of new care approaches may be enhanced.

2. Findings showed that older adults with $\mathrm{AD}$ and their caregivers have different views on aspects of robot care and functions. Both views need to be accounted for in a relationship-centered approach to dementia care. If an assistive robot is considered, it is critical to be mindful of the social needs and relationship dynamics of older adults and their caregivers. The choice of involving any technology in care needs to be individually determined by an older adult and his or her caregiver(s).

3. Implementation goals of assistive robots may be to enable older adults' autonomy and performance of daily activities, decrease frustration and stress of older adults and caregivers, and decrease caregiver burden without compromising social relationships. Long-term studies with assistive robots in the home setting will be necessary to demonstrate if these goals are achievable.

4. Participants expressed numerous possibilities for the development of robot functions for daily activities that are familiar or new, and simple to complex. The diversity of opportunities named, especially by caregivers, also means that the technology will need to be customizable.

5. Adaptability of the technology to changing needs of users with dementia and their caregivers will need to be considered to support acceptance and longevity with regards to robot use.

6. Participants' perceptions of the robot's role varied from companion to appliance. Researchers need to be cognizant of the roles that older adults and caregivers expect of robots and the possible relationships that users anticipate so that robots may be designed with the interfaces and functions to meet these expectations. It cannot be assumed that older adults want robots to be their friends.

7. Some older adult participants alluded to the stigma associated with using technology intended for older people with whom they did not identify. The implications of stigma on nonacceptance of potentially beneficial technology need to be considered by developers, researchers, and clinicians. Some of these issues may be addressed by designing technology that may be usable by everyone and customizable for those who have specific needs. Robots that may be usable by the whole family may be more acceptable and clinicians may introduce technologies earlier such that older adults may have the opportunity to become familiar with a robot that supports future needs.

\section{Limitations}

This study has multiple limitations. The 10 dyads who participated may not be representative of the population of older adults with mild-to-moderate $\mathrm{AD}$ and their caregivers. There is potential for volunteer bias as people who are more open to 
participating in research or to technology such as robots may be more likely to participate. While efforts were made to recruit from diverse sources, all participants came from one memory clinic. However, participants varied with respect to age, cognitive levels, abilities, symptoms, and care needs for older adults, living situations, and relationship statuses. Transferability of the findings to other older adults with $\mathrm{AD}$ and their caregivers needs to consider the backgrounds of the participants. The unfamiliar environment, novelty of robot use, and degree of stimulation from being in the study may influence how participants perceived the robot interaction. Participants interacted or observed an interaction with a tele-operated robot prototype so it may be challenging for them to imagine how an autonomous robot might function in the future. This may limit the possibilities of how the robot is perceived. Older adults' memory impairment and recall bias may make it difficult to get reliable accounts of their experiences. However, even if short-term memory for events was impaired, they would be able to express their overall impressions or affective responses to the robot. Including the perspectives of caregiver observers greatly adds to the interpretation of the findings. There is also potential for social desirability bias, where participants respond in a way they believe they will be perceived more positively. Researchers encouraged participants to respond critically and stressed that the robot was a prototype and that feedback would be applied to make improvements. Participants were exposed to the robot for only a short time so factors such as novelty may have impacted participants' responses. Long term studies in real home settings are necessary. Studies with a larger number of dyads are needed to explore patterns of responses to robot care, for example with respect to relationships between openness to robot care and the severity of dementia or the living situation of older adults (e.g., living alone or with a spouse).

\section{Conclusions}

The study addresses several important gaps in the development and implementation of assistive robots for older adults with mild-to-moderate $\mathrm{AD}$ and their caregivers. Through interviews following robot interactions, in-depth perspectives were solicited on robots that help with stepwise prompting for daily activities. Few projects have included older adults with this degree of cognitive impairment in the development of robots that assist with daily activities at home. Older adults in the study did not feel they needed such a robot at the time of the study, but felt an assistive robot could be useful for a range of daily activities for those who need the help. Most were open to the idea in the future. Caregivers were more open to an assistive robot and identified a broad range of activities and ways in which a robot could help. Several caregivers would like to have a robot at home at the time of the study. The introduction of an assistive robot to a caregiving scenario could have positive and negative consequences including decreased frustration and stress on both older adults and caregivers resulting in decreased relationship strain, increased social interaction for older adults if they felt the robot could be a companion, or decreased social interaction with caregivers. Ongoing dialogue between users and developers and careful consideration of robot design and caregiving relationship factors are necessary to achieve the potential of assistive robots.

\section{Conflict of interest}

None.

\section{Description of authors' roles}

R.H. Wang formulated the research questions, designed the study, collected the data, analyzed the data, and wrote the paper. A. Sudhama analyzed the data and assisted with writing the paper. $M$. Begum collected the data and reviewed the data analysis and the paper. R. Huq developed the teleoperated robot used in the study, assisted with collecting the data, and reviewed the data analysis and the paper. A. Mihailidis obtained the funding and supervised the study, assisted with formulating the research questions, assisted with designing the study, and reviewed the data analysis and the paper.

\section{Acknowledgments}

The authors wish to thank all the participants who were involved in this study and gratefully acknowledge the assistance of Drs. Jennifer Boger, Goldie Nejat, and Sharon Cohen. Funding for the study was provided by a Canadian Institutes of Health Research Catalyst Grant: Pilot Projects in Aging (201009). Funding for R.H. Wang was provided by a Canadian Institutes of Health Research Postdoctoral Fellowship.

\section{References}

American Psychiatric Association (2013). Neurocognitive disorders. In American Psychiatric Association (ed.), 
Diagnostic and Statistical Manual of Mental Disorders, 5th edn. Arlington, VA, USA: American Psychiatric Publishing.

Bedaf, S., Gelderblom, G. J. and De Witte, L. (2015). Overview and categorization of robots supporting independent living of elderly people: what activities do they support and how far have they developed. Assistive Technology, 27, 88-100. doi: 10.1080/10400435.2014.978916.

Bewernitz, M. W., Mann, W. C., Dasler, P. and Belchior, P. (2009). Feasibility of machine-based prompting to assist persons with dementia. Assistive Technology, 21, 196-207. doi: 10.1080/10400430903246050.

Braun, V. and Clarke, V. (2006). Using thematic analysis in psychology. Qualitative Research in Psychology, 3, 77-101. doi: 10.1191/1478088706qp063oa.

Broadbent, E., Tamagawa, R., Patience, A. and Knock, B. (2012). Attitudes towards health-care robots in a retirement village. Australasian fournal on Ageing, 31, 115-120. doi: 10.1111/j.1741-6612.2011.00551.x.

Cohen, C., Colantonio, A. and Vernich, L. (2002). Positive aspects of caregiving: rounding out the caregiver experience. International fournal of Geriatric Psychiatry 17, 184-188. doi: 10.1002/gps.561.

Fasola, J. and Matarić, M. J. (2013). A socially assistive robot exercise coach for the elderly. Fournal of Human-Robot Interaction, 2, 3-32. doi: 10.5898/JHRI.2.2.Fasola.

Faucounau, V. et al. (2009). Caregivers' requirements for in-home robotic agent for supporting community-living elderly subjects with cognitive impairment. Technology and Health Care, 17, 33-40. doi: 10.3233/THC-2009-0537.

Folstein, M., Folstein, S. E. and Mchugh, P. R. (1975). "Mini-mental state" a practical method for grading the cognitive state of patients for the clinician. Fournal of Psychiatric Research, 12, 189-198.

Frennert, S. and Ostlund, B. (2014). Review: seven matters of concern of social robots and older people. International Fournal of Social Robotics, 6, 299-310. doi: 10.1007/s12369-013-0225-8.

Jennings, L. et al. (2015). Unmet needs of caregivers of individuals referred to a dementia care program. The American Geriatrics Society, 63, 282-289. doi: 10.1111 /jgs. 13251.

Kales, H. C., Gitlin, L. N. and Lyketsos, C. G. (2015). Assessment and management of behavioral and psychological symptoms of dementia. British Medical fournal, 350, 1-16. doi: 10.1136/bmj.h369.

Kerssens, C. et al. (2015). Personalized technology to support older adults with and without cognitive impairment living at home. American fournal of Alzheimer's Disease and Other Dementias, 30, 85-97. doi: $10.1177 / 1533317514568338$.

Louie, W.-Y., Mccoll, D. and Nejat, G. (2014). Acceptance and attitudes toward a human-like socially assistive robot by older adults. Assistive Technology, 26, 140-150. doi: 10.1080/10400435.2013.869703

Mann, J. A., Macdonald, B. A., Kuo, I.-H., Li, X. and Broadbent, E. (2015). People respond better to robots than computer tablets delivering healthcare instructions. Computers in Human Behavior 43, 112-117. doi: 10.1016/j.chb.2014.10.029.

Mihailidis, A., Boger, J., Canido, M. and Hoey, J. (2007). The use of an intelligent prompting system for people with dementia: a case study. ACM Interactions (Special issue on Designing for seniors: innovations for graying times), 14, 34-37. doi: 10.1145/1273961.1273982.

Mihailidis, A., Boger, J., Craig, T. and Hoey, J. (2008). The coach prompting system to assist older adults with dementia through handwashing: an efficacy study. $B M C$ Geriatrics, 8. doi:10.1186/1471-2318-8-28.

Mitzner, T. L. et al. (2010). Older adults talk technology: technology usage and attitudes. Computers in Human Behavior, 26, 1710-1721. doi: 10.1016/j.chb.2010.06.020.

Moyle, W., Jones, C., Cooke, M., O'dwyer, S., Sung, B. and Drummond, S. (2014). Connecting the person with dementia and family: a feasibility study of a telepresence robot. BMC Geriatrics, 14, 1-11. doi: 10.1186/1471-2318-14-7.

Moyle, W., Kellett, U., Ballantyne, A. and Gracia, N. (2011). Dementia and loneliness: an Australian perspective. Fournal of Clinical Nursing, 20, 1445-1453. doi: 10.1111/j.1365-2702.2010.03549.x.

Nielsen, J. and Landauer, T. (1993). A mathematical model of the finding of usability problems. In INTERACT '93 and CHI '93 Conference on Human Factors in Computing Systems, April 24-29, 1993, Amsterdam, the Netherlands. New York: ACM, pp. 206-213.

Papastavrou, E., Kalokerinou, A., Papacostas, S. S., Tsangari, H. and Sourtzi, P. (2007). Caring for a relative with dementia: family caregiver burden. Fournal of Advanced Nursing, 58, 446-457. doi: 10.1111/j.1365-2648.2007.04250.x.

Peacock, S. et al. (2010). The positive aspects of the caregiving journey with dementia: using a strengths-based perspective to reveal opportunities. Fournal of Applied Gerontology, 29, 640-659. doi: $10.1177 / 0733464809341471$.

Richardson, T., Lee, S., Berg-Weger, M. and Grossberg, G. (2013). Caregiver health: health of caregivers of Alzeimer's and other dementia patients. Current Psychiatry Reports, 15, 1-7. doi: 10.1007/s11920-013-0367-2.

Rosenberg, L., Kottorp, A. and Nygard, L. (2012). Readiness for technology use with people with dementia: the perspectives of significant others. Fournal of Applied Gerontology, 31, 510-530. doi: $10.1177 / 0733464810396873$.

Sakuae, M. and Reid, D. (2012). Making tea in place: experiences of women engaged in a Japanese tea ceremony. Fournal of Occupational Science, 19, 283-291. doi: 10.1080/14427591.2011.610775.

Samsi, K. and Manthrope, J. (2013). Everyday decision-making in dementia: findings from a longitudinal interview study of people with dementia and family carers. International Psychogeriatrics, 25, 949-961. doi: 10.1017/S1041610213000306.

Saumure, K. and Given, L. (2008). Rigor in qualitative research. In L. M. Given (ed.), The Sage Encyclopedia of Qualitative Research Methods. Thousand Oaks, CA, USA: SAGE Publications, Inc.

Saunders, J. and Byrne, M. (2002). A thematic analysis of families living with schizophrenia. Archives of Psychiatric Nursing, 16, 217-223. doi: 10.1053/apnu.2002.36234.

Schroeter, C. et al. (2013). Realization and user evaluation of a companion robot for people with mild cognitive impairments. In IEEE International Conference on Robotics 
and Automation (ICRA 2013). Karlsruhe, Germany: IEEE, pp. 1145-1151.

Sharkey, A. and Sharkey, N. (2012). Granny and the robots: ethical issues in robot care for the elderly. Ethics and Information Technology, 14, 27-40. doi: 10.1007/s 10676-010-9234-6.

Shibata, T. (2012). Therapeutic seal robot as biofeedback medical device: qualitative and quantitative evaluations of robot therapy in dementia care. Proceedings of the IEEE, 100, 2527-2538. doi: 10.1109/JPROC.2012.2200559.

Smarr, C.-A. et al. (2014). Domestic robots for older adults: attitudes, preferences, and potential. International fournal of Social Robotics, 6, 229-247. doi: 10.1007/s12369-013-0220-0.
Topo, P. (2009). Technology studies to meet the needs of people with dementia and their caregivers. Fournal of Applied Gerontology, 28, 5-37. doi: 10.1177/0733464808324019.

Vallor, S. (2011). Carebots and caregivers: sustaining the ethical ideal of care in the twenty-first century. Philosophy and Technology, 24, 251-268. doi: 10.1007/s13347-011-0015-x.

Wu, Y.-H., Cristancho-Lacroix, V., Fassert, C., Faucounau, V., De Rotrou, J. and Rigaud, A.-S. (2016). The attitudes and perceptions of older adults with mild cognitive impairment toward an assistive robot. fournal of Applied Gerontology, 35, 3-17. doi: 10.1177/0733464813515092. 